\title{
A multiple criteria facility layout problem using data envelopment analysis
}

\author{
Najmeh Bozorgi $^{\mathrm{a}^{*}}$ and Mostafa Abedzadeh ${ }^{\mathrm{a}}$
}

${ }^{a}$ Department of Industrial Engineering, K. N. Toosi University of Technology, Tehran, 143344 Iran

\begin{tabular}{l}
\hline A R T I C L E I N F O \\
\hline Article history: \\
Received December 102010 \\
Received in revised form \\
1 February 2011 \\
Accepted 3 February 2011 \\
Available online \\
3 February 2011 \\
\hline Keywords: \\
DEA \\
Multi-criteria layout planning \\
Data envelopment analysis \\
Tabu search \\
Dynamic tabu list \\
Efficiency
\end{tabular}
A B S T R A C T

\begin{abstract}
In this paper, we propose a multi criteria decision making technique to determine an efficient solution for quadratic assignment problem. The proposed method of this paper considers transportation cost, adjacency and separation as the most important criteria to find the efficient layout. A tabu search is used to generate a set of feasible solutions and for each solution, we evaluate various criteria. The proposed model of the paper uses DEA technique to choose the most efficient units among the feasible solutions. The implementation of the proposed method is demonstrated using some benchmark problems and the results are discussed in details.
\end{abstract}

(c) 2010 Growing Science Ltd. All rights reserved.

\section{Introduction}

The static facility layout problem is the determination of the most efficient arrangement of departments within a facility. There are different examples of layout facilities such as manufacturing plants, administrative office buildings, service facilities, etc. The efficient arrangement of resources (e.g. machines, departments, or workforce) within the facility results in a well-coordinated workflow among the resources. An efficient layout helps other operations, which are dependent on workflow, to perform well (Mckendall et al, 2006).

For manufacturing facilities, material-handling cost is the most significant measure to determine the efficiency of a layout. Material-handling cost represents $20-50 \%$ of the total operating cost and 15$70 \%$ of the total cost of manufacturing a product (Tompkins et al., 1996). The Material handling cost is determined based on the flows and distances of materials among various departments.

Facility layout problems (FLPs) can be classified in two kinds of problems of discrete layout problems (DLPs) and continuous layout problems (CLPs). DLP divides the plant site into many rectangular blocks. Each block has the same area and shape, and each block is assigned to a facility. If the facilities have unequal areas, they could occupy blocks and they are modeled into a cell. 
Quadratic assignment problem (QAP) is the most famous one for discrete layout problems and the proposed model of this paper uses this method as part of problem statement. In CLP, all facilities may be placed anywhere within the planning site, and the facilities must not overlap each other (Wang et al., 2005). Furthermore, FLPs can be further categorized according to the type of material flows. The nature of flowing materials among departments can be either deterministic or stochastic. Deterministic flow data are fixed and known with certainty. In contrast, when the material flows are not known with certainty, the flow is a random variable and may be represented as a probability distribution. This type of material flow data is defined as stochastic (Liu, 2005).

There are also some cases where the problem is modeled as multi-objective mathematical programming. In such as case, instead of looking for an optimal solution(s) we look for Paretooptimal solution(s). There are different multi-objective techniques such as Lp-Norm, Lexicography, Goal programming, DEA, etc. DEA has been widely used to measure the relative efficiency of different similar units. DEA can also be used to select an efficient solution from various efficient solutions. For the case of DLP problem, an efficient solution based on different criteria can be chosen when there are different alternatives. The proposed model of this paper considers different criteria to be used as input/output parameters needed for the implementation of DEA method.

This organization of this paper first presents the literature review. The problem statement and the solution procedure are explained in section 3 and the concluding remarks are given in section 4 to summarize the contribution of the paper.

\section{Literature survey}

Measuring the relative efficiency of a system has been an important task in management for the purpose of control, planning, etc. (Bashiri \& Dehghan, 2010). Data envelopment analysis (DEA) is one of the most popular techniques to use to measure the relative efficiency of different units when there is more than one input/output. Charnes et al. (1978) are the first people who introduced DEA technique and Banker et al. (1984) contributed to the model for variable return to scale problems. DEA, a non-parametric linear programming based method, has gained a wide range of applications measuring comparative efficiency of multiple inputs and outputs of a homogeneous set of decision making units (DMUs). One reason is that DEA has opened up the possibilities to use the method for the cases where the other approaches cannot handle very easily (Cooper et al, 2006). There are literally different types of DEA methods used for various purposes such as fuzzy DEA, robust DEA, interval DEA, etc (Amin \& Toloo, 2007). The minmax DEA model is one of the popular techniques, which could be used to calculate the relative efficiency and it has the following problem statement,

$$
\begin{aligned}
& \text { (Minmax DEA) } \min z=\widetilde{\zeta} \\
& \text { subject to } \\
& \sum_{i=1}^{I} v_{i} \phi_{i .}=1, \\
& \sum_{r=1}^{s} u_{r} \varphi_{r j}-\sum_{i=1}^{m} v_{i} \phi_{i j}+\zeta_{j}=0, \\
& \\
& \hat{\zeta}^{\prime}-\zeta_{j} \geq 0 \quad(j=1,2, \cdots n), \\
& u_{r}, v_{j}, \zeta_{j} \geq 0
\end{aligned}
$$

where $j$ is the number of DMUs, $r$ is the number of outputs $(r=1, \cdots s)$ and $i$ is the number of inputs $(i=1, \cdots m)$. Also $u_{r}$ and $v_{j}$ are the weights of $r$ th output and $i$ th input, respectively. $\varphi_{r j}$ the $r$ th output for $j$ th DMU, $\phi_{i j}$ is the $i$ th input for the $j$ th DMU. $\zeta_{j}$ is defined as inefficiency of DMUs, $\hat{\zeta}$ is the maximum inefficiency. Koopmans and Beckmann (1957) were the first to model the FLP with equal size departments, as a QAP. They considered profit associated with each department to location assignment. Furthermore, there is a flow of commodity among pairs of the departments. Gilmore 
(1962) and Lawler (1963) were the first to develop optimal procedures for the QAP problem defined by Koopmans and Beckmann (1957) based on branch and bound techniques. Armour and Buffa (1963) developed a pairwise exchange improvement type heuristic to solve the FLP with unequal area departments. Buffa et al. (1964) developed an improvement type heuristic called computerized relative allocation of facilities technique (CRAFT), to solve the FLP with unequal area departments. Bazaraa (1975) formulated the unequal area FLP using the discrete representation as a quadratic setcovering problem, developed a branch and bound algorithm to solve the resulted problem. Burkard and Rendl (1984) as well as Wilhelm and Ward (1987) applied simulated annealing (SA) heuristic to solve the QAP. Hassan et al. (1986) developed a construction heuristic, called SHAPE, to solve the discrete representation of the unequal area FLP. Li and Mashford (1990) applied a genetic search algorithm to solve the QAP. Skorin-Kapov (1990) was the first who applied a tabu search (TS) improvement type heuristic to the QAP. Chiang and Chiang (1998) applied simulated annealing, tabu search, probabilistic tabu search (PTS), a hybrid of tabu search and simulated annealing heuristics to solve the QAP. Moreover, Drezner (2008) used a memetic algorithm (MA) to solve the QAP. The MA was developed by Norman and Moscato (1989) and is a hybrid of GA and TS. Tam (1992-a) developed a GA to solve the FLP with unequal area variable shape departments and this problem was developed by Tam (1992-b) and Tate and Smith (1995) using SA and GA. The authors used the flexbay structure, developed by Tong (1991), to construct the layout. Imam et al. (2000) presented an MILP formulation and developed a two phase heuristic to solve the FLP with unequal area fixed shape departments. Dunker et al. (2003) used a GA to solve the FLP with unequal area fixed shape departments. The shapes of the departments are fixed and the heuristic considers pick-up/drop-off points. . Kochhar et al. (1997) outlined a GA based algorithm to solve the single-floor facility layout problem and they considered both equal and unequal sizes. Chwif et al. (2001) presented a hybrid optimization approach for the layout design of unequal-area facilities. Scholz et al. (2009) applied a slicing tree based tabu search heuristic for the rectangular, continual plane facility layout problem (FLP). Komarudin and Wong (2009) were the first to apply ant system (one of the ACO variants) to solve the unequal layout problem. Ghosh et al. (2011) performed a comprehensive study on the implementation of meta-heuristic approaches on layout problems. Also, Jabal-Ameli et al. (2011) presented heuristic methods to solve capacitated location-routing problems.

There are also some cases where other decision-making techniques are used for layout planning. For example, Yang and Kou (2003) proposed a hierarchical analytic hierarchy process (AHP) and DEA approach to solve FLP. A computer-aided layout-planning tool was used to generate a considerable number of layout alternatives as well as to generate quantitative decision-making unit (DMU) outputs. The qualitative performance measures were weighted by AHP and DEA was then used to solve the multiple-objective layout problem. In addition, to design an efficient robust layout design, Ertay et al. (2006) used DEA to evaluate their layouts and considered different criteria as inputs and outputs for DEA to evaluate alternatives (layouts). They considered transportation cost and adjacency score as input and shape ratio, flexibility, quality and hand-carry utility as outputs. Furthermore, Bashiri and Dehghan (2010) considered different criteria in addition to cost to suggest an efficient solution for dynamic layout problem. They first used classical models for DLP1to generate a good solution from a cost point of view. Then they defined DMUs and their inputs and outputs based on the classic DLP solution and a multi-objective combined DLP -DEA model was proposed to solve the resulted problem.

\section{The proposed method}

In order to gain the most effective layout in this research, we use the implementation of the tabu search heuristic originally presented by Chiang and Kouvelis (1996). The method considers the diversity strategy and dynamic tabu list. First, an initial layout is generated, randomly. For instance; suppose we have four departments, if the first department is assigned to location 2, department 2 is assigned to location 1 and four departments are assigned similarity. After we produce an initial solution, quality and quantity criteria are calculated which includes the cost, required adjacency value 
and required distance value. Next, we create, in each iteration, the neighbourhoods for current solution inputs and outputs of each layout and the most efficient layout is chosen as the best answer. The resulted solution is considered as an initial solution for the next iteration. This process will go on until the algorithm reaches its stopping condition. The quality and quantity criteria will be respectably explained as follows.

\subsection{Neighborhood structure}

The local searched technique used in tabu search heuristic is the steepest descent pairwise exchange heuristic. The main idea of heuristic is to find the best solution of pairwise exchange in the neighbourhood of the current solution. Therefore, we consider all possible pairwise exchanges in each period and we define each replacement as a movement.

If we have $N$ departments, the number of movements in each period is equal to $C_{2}^{\mathrm{N}}=\frac{\mathrm{N}(\mathrm{N}-1)}{2}$. In this paper, in addition to pairwise exchange heuristic to create neighbourhood, we use another strategy named reverse strategy. In this strategy, in addition to exchange the center of two departments we reverse the center of department between them. The number of movement resulted from this strategy in each iteration is $C_{2}^{\mathrm{N}}=\frac{\mathrm{N}(\mathrm{N}-1)}{2}$. Therefore, the number of the whole movements in each period is $N(N-1)$. Next, the total cost and the value of adjacency and distance request will be calculated for each movement and the best admissible move is selected. The best admissible movement is the movement which is not tabu or its total cost is less than the total cost of preformed movements (aspiration criteria).

\subsection{TS Heuristic with diversification strategies}

We use a special form of diversity strategy, which includes the frequency-based memory, penalty function for non-improving movements and dynamic tabu list as a recent-based memory strategy.

\subsubsection{Frequency-based Memory}

The frequency-based memory structure is used to memorize the trace of repeated movement. Information is registered within the triangular at the bottom of the tabu array ( $\operatorname{tabu}[i][k]$ where $i>k$ ). The value of tabu[i][k], where $i>k$ stands for the number of times that the location of the departments $i$ and $k$ is chosen as the best expectable movement. Fig. 1 demonstrates an example of a tabu list where tabu[1][4]=1, which means that the departments 1 and 4 are exchanged once.

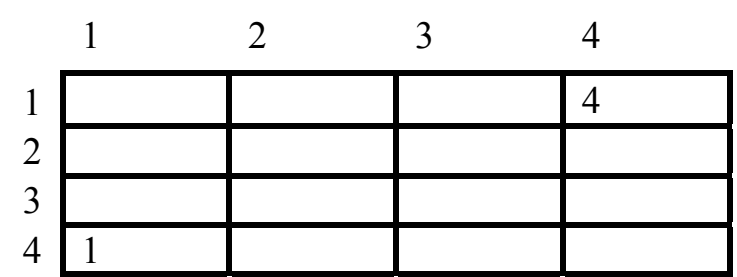

Fig. 1. The tabu list with frequency based memory

In addition, a penalty function is used to penalize non-improving movements. In other words, penalty function assigns a penalty to the repeated movements registered with in triangular at the bottom of the tabu array to penalize non-improving movement. Penalty function is the summation of the total cost of non-improving movements. If a movement is an improving one, it will not be penalized. Therefore, the penalty function that exchanges the location of the department, $i$ and $k$ can be defined as follows,

$\mathrm{p}(\mathrm{i}, \mathrm{k})=\left\{\begin{array}{lr}0 & \Delta \mathrm{TC}_{\mathrm{ik}}(\pi)>0\left(\mathrm{TC}\left(\pi^{\prime}\right)<T C(\pi)\right) \\ \mathrm{a} * \operatorname{tabu}[\mathrm{i}][\mathrm{k}] & \text { otherwise }\end{array}\right.$

where a is a penalty parameter.

3.2.2 Dynamic Tabu List Size 
The dynamic tabu list size (or tabu length) is a recently-based memory strategy which is applied to give variation to search space. The dynamic tabu length, $T L_{d}$, is a variable between a lower bound (LB) and an upper bound (UB). It changes from one iteration to another one depending on the amount of total cost reduction compared with the best admissible solution (TC $\left(\pi^{`}\right.$ best $)$ ).

Let $\mathrm{TC}(\pi)$ be the current solution, the relative cost reduction of $(\operatorname{PR}(\pi))$ is calculated as $P R(\pi)=\frac{T C(\pi)-T C\left(\pi_{\text {best }}^{\prime}\right)}{T C(\pi)} \times 100 \%$. Table 1 summarizes the details on how $T L_{d}$ is set.

Table 1

Dynamic Tabu list size

$\%$ Reduction of the total $\operatorname{cost}(\mathrm{PR}(\pi))$

$\begin{array}{cc}\geq \beta \% & T L \beta=2 N \\ \alpha \% \leq P R(\pi)<\beta \% & U B \\ 0 \% \leq P R(\pi) \leq \alpha \% & L B+(U B-L B) * P R(\pi) / \alpha \% \\ P R(\pi)<0 \% & \text { in the previous iteration } T L_{d}\end{array}$

\subsection{DEA input/outputs}

The implementation of the proposed DEA model of this paper uses one input, the cost of transportation, and two outputs, adjacency and separation. The most efficient unit(s) from the solution of the implementation of minmax DEA model determines the optimal layout facility.

\subsubsection{Cost}

The proposed DEA model of this paper has one input which is the cost of transportation calculated as the solution of the following quadratic assignment programming (QAP),

(QAP) $\min$

$$
T C=\sum_{i=1}^{N} \sum_{j=1}^{N} \sum_{k=1}^{N} \sum_{l=1}^{N} f_{i k} d_{j l} x_{i j} x_{k l}
$$

subject to

$$
\begin{aligned}
& \sum_{j=1}^{N} x_{i j}=1, i=1, \cdots, N \\
& \sum_{i=1}^{N} x_{i j}=1, j=1, \cdots, N \\
& x_{i j}=\{o, 1\}, i, j=1, \cdots N .
\end{aligned}
$$

According to QAP model, $x_{i j}=1$ if facility $i$ is assigned to department $j$ and $x_{i j}=0$, otherwise. Also $x_{k l}=1$ if facility $k$ is assigned to department $l$ and $x_{k l}=0$, otherwise, $f_{i k}$ and $d_{j l}$ are the flow and the distance between departments, respectively. The objective function (TC) minimizes the sum flow costs among various departments. Constraint set (6) ensures that each location is assigned to only one department and constraint set (7) ensures that only one department is assigned to each location.

\subsubsection{Adjacency}

Adjacency is another important factor which plays an important role on choosing the right place for a facility. Let $r_{i j}$ and $l_{i j t}$ be the closeness rating and the contact perimeter length between departments $i$ and $j$ in period $t$, respectively. According to Aiello et al. (2006), the adjacency ratio can be defined as follows,

$$
\text { Adjacency }=\sum_{i} \sum_{j} r_{i j} l_{i j t} \text {. }
$$




\subsubsection{Distance request}

There are also some cases where we want to separate two departments from each other for many reasons such as noise, vibration, etc. We may define a new measure called separation based on the distance and the degree of the separation as follows,

$$
\text { Seperation }=\sum_{i} \sum_{j} s_{i j} d_{i j t} \text {, }
$$

where $s_{i j}$ is the distance rating for departments $i$ and $j$ and $d_{i j t}$ is the distance between the centers of two departments using a pre-specified metric in period $t$.

\section{Numerical results}

The proposed tabu search heuristics were tested on data sets given by Lacksonen and Enscore (1993). The data set contains test problems with $6,12,20$, and 30 departments (i.e., $\mathrm{N}=6,12,20$, and 30) each with 3 and 5 periods (i.e., $\mathrm{T}=3$ and 5). Each of these problem instances includes four test problems. We use only the data sets for the first period $(\mathrm{T}=1)$ to calculate the cost (input) and we randomly generate other data sets needed for the outputs using a uniform distribution function from 1 to10. As explained earlier, the proposed tabu search method generates different alternatives with various costs. For each solution we compute the two output measures. Since there are one input and two inputs, we need at least 9 solutions to be used for the implementation of DEA method. The optimal solution of the proposed DEA model was run using the Minmax DEA approach explained earlier. In case there is more than one efficient solution we chose the one with the lower cost.

The proposed TS heuristics were coded on MATLAB programming language. The test problems were solved on $2.5 \mathrm{GHz}$ PC with $4 \mathrm{~GB}$ of memory. Table 2 shows the results of the values for the input (cost) and outputs (adjacency, distance requested) of the most effective chosen layout.

Table 2

The optimal input and output values for Lacksonen and Enscore data set

\begin{tabular}{|c|c|c|c|c|c|c|c|c|c|c|c|}
\hline \multicolumn{2}{|c|}{ Problem size } & \multirow{2}{*}{$\begin{array}{l}\text { Prob. } \\
\text { No. }\end{array}$} & \multirow{2}{*}{ cost } & \multirow{2}{*}{ Adjacency } & \multirow{2}{*}{ Separation } & \multicolumn{2}{|c|}{ Problem size } & \multirow{2}{*}{$\begin{array}{l}\text { Prob. } \\
\text { No. }\end{array}$} & \multirow{2}{*}{ cost } & \multirow{2}{*}{ Adjacency } & \multirow{2}{*}{ Separation } \\
\hline $\mathrm{N}$ & $\mathrm{T}$ & & & & & $\mathrm{N}$ & $\mathrm{T}$ & & & & \\
\hline \multirow{8}{*}{6} & \multirow{4}{*}{3} & P01 & 107 & 28 & 56.5028 & \multirow{8}{*}{20} & & P17 & 1132 & 76 & 857.017 \\
\hline & & P02 & 95 & 22 & 56.5028 & & \multirow{3}{*}{3} & P18 & 1995 & 58 & 822.9014 \\
\hline & & P03 & 113 & 28 & 56.5028 & & & P19 & 1096 & 102 & 788.6169 \\
\hline & & P04 & 85 & 30 & 56.5028 & & & $\mathrm{P} 20$ & 2104 & 112 & 765.5205 \\
\hline & \multirow{4}{*}{5} & P05 & 108 & 30 & 56.5028 & & \multirow{4}{*}{5} & P21 & 1053 & 60 & 869.3061 \\
\hline & & P06 & 79 & 30 & 56.5028 & & & P22 & 2039 & 136 & 711.7053 \\
\hline & & P07 & 107 & 28 & 56.5028 & & & $\mathrm{P} 23$ & 1046 & 78 & 807.3893 \\
\hline & & $\mathrm{P} 08$ & 95 & 22 & 56.5028 & & & $\mathrm{P} 24$ & 2136 & 132 & 923.8916 \\
\hline \multirow{8}{*}{12} & \multirow{4}{*}{3} & P09 & 650 & 38 & 210.8138 & \multirow{8}{*}{30} & \multirow{4}{*}{3} & P25 & 7327 & 102 & 1882.824 \\
\hline & & $\mathrm{P} 10$ & 737 & 32 & 213.6641 & & & P26 & 5504 & 188 & 1920.948 \\
\hline & & P11 & 595 & 10 & 199.8713 & & & P27 & 2688 & 158 & 2065.375 \\
\hline & & $\mathrm{P} 12$ & 706 & 28 & 192.813 & & & P28 & 5445 & 210 & 1857.455 \\
\hline & \multirow{4}{*}{5} & P13 & 640 & 24 & 211.4561 & & \multirow{4}{*}{5} & P29 & 2688 & 176 & 2073.372 \\
\hline & & P14 & 720 & 14 & 210.424 & & & P30 & 5399 & 244 & 2122.551 \\
\hline & & $\mathrm{P} 15$ & 657 & 24 & 197.249 & & & P31 & 2724 & 182 & 2294.372 \\
\hline & & $\mathrm{P} 16$ & 737 & 32 & 213.6641 & & & $\mathrm{P} 32$ & 5585 & 230 & 2100.201 \\
\hline
\end{tabular}

The values of the costs for the proposed model reported in Table 2 are slightly more than the resulted values for the same problems by considering only the cost, but we have gained the most efficient arrangement considering the other criteria. The resulted layout using the proposed model of this paper seems to be more applicable for real-world problems. On the other hand, the multi-objective approach 
is considered for the classical approach to the facility layout problems. We have presented one layout resulted out from the proposed approach as an instance in Fig 2.

\begin{tabular}{|l|l|l|l|}
\hline 9 & 20 & 4 & 15 \\
\hline 11 & 12 & 17 & 14 \\
\hline 18 & 1 & 5 & 10 \\
\hline 2 & 19 & 13 & 16 \\
\hline 3 & 8 & 6 & 7 \\
\hline
\end{tabular}

Fig. 2. Efficient solution for a FLP with 20 departments (P22)

\section{Conclusion}

In this paper, we have presented a multi criteria decision making to determine an efficient solution for quadratic assignment problem. The proposed method of this paper considered transportation cost, adjacency and separation as the most important criteria to find the efficient layout. A tabu search was also implemented to generate a set of feasible solutions and for each solution, all three criteria were measured. The proposed model of the paper adopted a minmax DEA technique to choose the most efficient units among the feasible solutions and in case there was more than one single efficient solution, the one with the lowest cost was selected. This paper can be extended using other metaheuristic techniques such as particle swarm optimization, firefly, etc and we leave it as a future research.

\section{References}

Aiello, G., Enea, M., \& Galante, G. (2006). A multi-objective approach to facility layout problem by genetic search algorithm and ELECTERE method. Robotics and Computer-Integrated Manufacturing, 22,447455.

Amin, Gh R., \& Toloo, M. (2007). Finding the most efficient DMUs in DEA: An improved integrated model. Computers \& Industrial Engineering, 52, 71-77.

Armour, G. C., \& Buffa, E. S. (1963). A heuristic algorithm and simulation approach to relative location of facilities. Management Science, 9, 294-309.

Buffa, E. S., Armour, G. C., \& Vollman, T. E. (1964). Allocating facilities with CRAFT, Harvard Business Review, 42, 136-158.

Banker, R. D., Charnes, A., \& Cooper, W. W. (1984). Some models for estimating technical and scale inefficiency in data envelopment analysis. Management Science, 30, 1078-1092.

Bashiri, M., \& Dehghan, E. (2010). Optimizing a multiple criteria dynamic layout problem using a simultaneous data envelopment analysis modeling Optimizing a DLP using DEA. International Journal on Computer Science and Engineering, 2(1), 28-35.

Bazaraa, M. S. (1975). Computerized layout design: a branch and bound approach. AIIE Transactions, 7, 432438.

Burkard, R. E., \& Rendl, F. (1984). A thermodynamically motivated simulation procedure for combinatorial optimization problems. European Journal of Operational Research, 17, 169-174.

Charnes, A., Cooper, W. W., \& Rhodes, E. (1978). Measuring the efficiency of decision-making units. European Journal of Operational Research, 2, 429-444.

Chiang, W., \& Chiang, C. (1998). Intelligent local search strategies for solving facility layout problems with the quadratic assignment problem formulation. European Journal of Operational Research, 106, 457-488.

Chiang, W., \& Kouvelis, P. (1996). An improved Tabu search heuristic for solving facility layout design problems. International Journal of Production Research, 34(9), 2565-2585.

Chwif, L., Barretto, M. R. P., \& Moscato, L. A. (1998). A Solution to the Facility Layout Problem Using Simulated Annealing. Computer In Industry, 36, 125-132.

Cooper, W. W., Seiford, L., \& Tone, M. K. (2006). Introduction to Data Envelopment Analysis and its uses with DEA-Solver software and references. Springer.

Drezner, Z. (2008). Extensive experiments with hybrid genetic algorithms for the solution of the quadratic assignment problem. Computers and Operations Research, 35, 717-736. 
Dunker, T., Radons, G., \& Westkamper, E. (2003). A coevolutionary algorithm for a facility layout problem. International. Journal of Production Research, 15, 3479-3500.

Ertay, T., Ruan, Da., \& Tuzkaya, U. R. (2006). Integrating data envelopment analysis and analytic hierarchy for the facility layout design in manufacturing systems. Information Sciences, 176, 237-262.

Gilmore, P. C. (1962). Optimal and suboptimal algorithms for the quadratic assignment problem. Journal of the Society for Industrial and Applied Mathematics, 10, 305-313.

Ghosh, T., Sengupta, S., Chattopadhyay, M., \& Dan, P. K. (2011). Meta-heuristics in cellular manufacturing: A state-of-the-art review. International Journal of Industrial Engineering Computations, 2(1), 87-122.

Hakobyan, A. (2008). Heuristic for Dynamic Facility Layout Problem with Unequal Area Problem. PhD Dissertation, Morganton, West Virginia.

Hassan, M. M. D., Hogg, G. L., \& Smith, D. R. (1986). SHAPE: a construction algorithm for area placement evaluation. International Journal of Production Research, 24, 1283-1295.

Jabal-Ameli, M. S., Aryanezhad, M. B., \& Ghaffari-Nasab, N. (2011). A variable neighborhood descent based heuristic to solve the capacitated location-routing problem. International Journal of Industrial Engineering Computations, 2(1), 141-154.

Kochhar, J. S., Foster, B. T., \& Heragu, S. S. (1997). A Genetic Algorithm for the Unequal Area Facility Layout Problem. Computers and Operations Research, 25, 583-594.

Komarudin, \& Wong, K.Y. (2010). Applying Ant System for Solving Unequal Area Facility Layout Problems. European Journal of Operational Research, 202, 730-746.

Koopmans, T. C., \& Beckmann, M. J. (1957). Assignment problems and the location of economic activities. Econometrica, 25, 53-76.

Kuppusamy, S. (2001). Simulated Annealing Heuristic for the Dynamic Facility Layout Problem. MS Dissertation, Morganton, West Virginia.

Lacksonen, T. A., \& Enscore E. E. (1993). Quadratic assignment algorithms for the dynamic layout problems. International Journal of Production Research, 31, 503-517.

Li, T., \& Mashford J. (1990). A parallel genetic algorithm for quadratic assignment Proceedings of the ISMM. International Conference Parallel and Distributed Computing and Systems, 391-394.

Liu, W. H. (2005). Tabu Search Heuristic for the Dynamic Facility Layout problem. MS Dissertation, Morganton, West Virginia.

Mckendal, A. R., Shang, J., \& Kuppusamy, S. (2006). Simulated annealing heuristics for the dynamic facility layout problem. Computers \& Operations Research, 33, 2431-2444.

Mir, M., \& Imam, M. H. (2001). A hybrid optimization approach for layout design of unequal area facilities. Computers \& Operations Research, 39, 49-63.

Norman, M. G., \& Moscato, P. (1989). A competitive and cooperative approach to complex combinatorial search. Caltech Concurrent Computation Program, Report 826.

Scholz, D. A., Petrick, \& Domschke, W. (2009). A slicing tree and tabu search based heuristic for the unequal area facility layout problem. European Journal of Operational Research, 197, 166-178.

Skorin-Kapov, J. (1990). Tabu search applied to the quadratic assignment problem. ORSA Journal on Computing, 2, 33-45.

Tam, K. Y. (1992). Genetic algorithms function optimization and facility layout design. European Journal of Operational Research 63: 322-346.

Tam, K. Y. (1992). A simulated annealing algorithm for allocating space to manufacturing cells. International Journal of Production Research, 30: 63-87.

Tate, D. E., \& Smith, A. E. (1995). A genetic approach to the quadratic assignment problem. Computers and Operations Research, 22, 73-83.

Tompkins, J. A.,White, J. A., Bozer, Y. A., Frazelle, E. H., Tanchoco, J. M. A., \& Trevino, J. (1996). Facilities planning. New York: Wiley; 137-285.

Tong, X. (1991). SECOT: a sequential construction technique for facility design, Unpublished Doctoral Dissertation, University of Pittsburgh, Pittsburgh, PA, USA.

Wang, M. J., Hu, M. H.,\& Ku, M. Y. (2005). A solution to the unequal area facilities layout problem by genetic algorithm. Computers In Industry, 56, 207-220.

Wilhelm, M. R., Ward, T. L. (1987). Solving the quadratic assignment problem by simulated annealing. IIE Transactions, 19, 107-119.

Yang, T., \& Kuo, Ch. (2003). A hierarchical AHP/DEA methodology for the facilities layout design problem. European Journal of Operational Research, 147, 128-136. 